\title{
José Gestoso y Pérez. Teoría y Praxis de la Conservación
}

\author{
Ma Dolores Ruiz de Lacanal Ruiz-Mateos
}

\begin{abstract}
Resumen: El objetivo de este artículo es hacer una reflexión sobre las aportaciones que José Gestoso y Pérez (1852-1917) realiza a la teoría y la praxis de la conservación. Persona de gran prestigio y mérito, desarrolló su labor profesional en Sevilla a mediados del siglo XIX y principios del siglo XX y ha sido objeto de numerosas investigaciones.

Tras la lectura de los estudios, que le resaltan como un referente claro de la cultura sevillana en sus múltiples manifestaciones, (Historia y crítica del Arte, Arqueología, Archivística, etc.), este artículo concentra la atención en sus propios textos, para descubrir que constituyen un conjunto coherente y estructurado de conocimientos teóricos y prácticos, aportan unos objetivos definidos y una metodología con recursos y estrategias, que son claros antecedentes de nuestros actuales "planes" de conservación.
\end{abstract}

Palabras claves: José Gestoso y Pérez, Conservación, Restauración, Teoría, Historia, Museo, Archivística, Patrimonio Cultural, siglo XIX y XX.

\section{José Gestoso y Perez. Teoria e prática da conservação}

Resumo: O objetivo deste artigo é refletir sobre as contribuições que José Gestoso y Pérez (1852-1917) proporcionou à teoria e à prática da conservação. Pessoa de grande prestígio e mérito, desenvolveu a sua carreira profissional em Sevilha, em meados do século XIX e início do século XX e tem sido objeto de inúmeras investigações.

Após a leitura dos estudos, que o destacam como uma referência clara da cultura sevilhana nas suas múltiplas manifestações (História e Crítica de Arte, Arqueologia, Arquivística, etc), este artigo foca a sua atenção nos seus próprios escritos, para descobrir que constituem um conjunto coerente e estruturado de conhecimentos teóricos e práticos, com uns objetivos definidos e uma metodología com recursos e estratégias, que são claros antecedentes dos nossos atuais "planos" de conservação.

Palavras-chave: José Gestoso y Pérez, Conservação, Restauro, Teoria, História, Museu, Arquivística, Património Cultural, séculos XIX e XX.

\section{José Gestoso y Perez. Theory and praxis of conservation}

Abstract: The aim of this paper is to reflect upon the contributions made by José Gestoso y Pérez (18521917) to the theory and praxis of the Conservation.

As a highly recognized and meritorious person, he developed his professional career in Seville in the middle of the nineteenth century and beginning of the twentieth century, and he has been the focus of many researches.

After reading the studies, that highlighted him as an evident reference of sevillian culture on its multiple expressions (History and Art Critic, Archeology, Archivist, etc.), this paper focus its attention on his own writings, to reveal that they constitute a coherent and structured foundation of practical and theoretical knowledge, with well-established objectives and a methodology with resources and strategies, which, clearly form, the precursors of our Conservation plans.

Keywords: José Gestoso y Pérez, Conservation, Restoration, Theory, History, Museum, Archive, Cultural Heritage, 19 and 20 centuries. 
"Tal es la magia de las bibliotecas: todo reposa como perlas y corales en el fondo de los estantes, pero nada muere jamás por completo, todo está a la espera de ser reconocido, releído un día para un nuevo valor uso".

Aby Warburg (Didi_Hubermann, 2009: 465).

\section{Introducción}

José Gestoso y Pérez, una de las personalidades más destacadas de la cultura sevillana de su tiempo, estuvo muy vinculado a las principales instituciones del momento. Entre ellas, al Museo de Bellas Artes, al que dedicó parte de tu trabajo y esfuerzo (López Rodríguez 2010: 288).

Para el museo redactó un primer catálogo de 1897 y otro posterior editado en 1912, de gran rigor y contenido científico. Miembro de la Comisión a la que se le encomendó la ubicación de las salas del museo, fue responsable de su museografía y su benefactor, ya que años después a su fallecimiento, su viuda, María Daguerre, donaría la colección del reconocido arqueólogo e historiador, formándose la Sala Gestoso, con objetos de arte, cerámica antigua y moderna, armas, azulejos y pinturas que le habían pertenecido. A esta colección pertenece el retrato de 1914 mostrado en el artículo, donde Gonzalo Bilbao, gran pintor y amigo, le retrata con el traje de ceremonia como académico.

Fue Académico Correspondiente de las Reales Academias Española, de San Fernando y de la Historia, Académico Secretario de Bellas Artes de Sevilla, Preeminente de la de Buenas Letras y Oficial de Instrucción Pública de Francia. Vinculado a la Academia de Bellas Artes, institución que tuvo a lo largo del siglo XIX la competencia en protección de los monumentos, formó parte también de la Comisión de Monumentos Históricos y Artísticos y ejerció su trabajo como archivero del Archivo Municipal de Sevilla (V.V.A.A. 2012: 25, 729; V.V.A.A. 1991:57).

Conservador del Museo de Arqueología de Sevilla, estuvo fuertemente vinculado a numerosos monumentos sevillanos, particularmente a la Catedral, cuya colección estudió, describió, catalogó y divulgó (López Rodríguez 2010: 131).
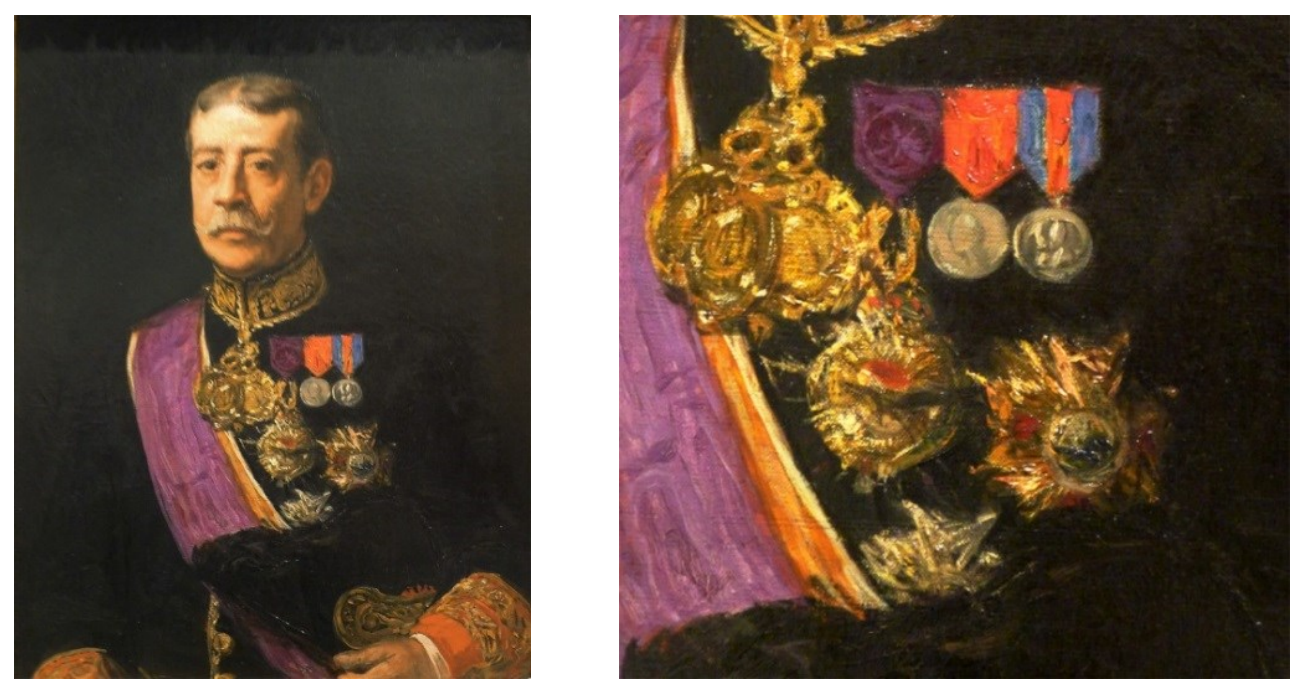

Figuras 1 y 2: Retrato de José Gestoso realizado por Gonzalo de Bilbao y detalle. Colección Museo de Bellas Artes de Sevilla. Fotografía realizada en la exposición de 2013. 


\section{La valoración de la obra de José Gestoso y Pérez}

Reconocido y valorado por un gran número de historiadores, críticos y arqueólogos, que se encargaron de resaltar su trabajo como dibujante, bibliófilo, arqueólogo, literato, ceramista, decorador, asesor de diversos procesos de restauración, archivero, coleccionista, mecenas y crítico del arte, el erudito sevillano, como prolífico escritor, con más de un centenar de trabajos publicados es una referencia indispensable en múltiples trabajos de Historia del Arte, Arqueología o Archivística. El estudio de su obra ha sido constante. Baste recordar el homenaje póstumo realizado por José Hernández Díaz (1946), que resaltaba su labor en la crítica del arte, las apreciaciones de Álvaro Recio Mir (2008), centradas en su concepto artístico, o el trabajo de Antonio José Albardonedo Freire (2010) valorando sus aportaciones a la historiografía del arte; a las que se sumarían entre otras, la mirada de José Beltrán Fortes (2002) a través de la Arqueología, o la de José Ramón López Rodríguez (2010), bajo el prisma de la historia de los museos de Andalucía; las investigaciones de Alfonso Pleguezuelo Hernández (1995) atentas especialmente al estudio del coleccionismo y el mecenazgo, y más concretamente al campo de la cerámica, y a Pedro Gómez Ramos, con su visión general que abunda en el papel que ocupa en la propia historia de Sevilla y su provincia. Todos contribuyeron a trazar su personalidad, guardar su memoria y retener para el recuerdo la enorme e interesante tarea que llevó a cabo a lo largo de su vida.

Sin embargo, sus publicaciones y sus documentos no se agotan y permiten ser valorados de nuevo, ahora bajo un nuevo valor, la disciplina de la Conservación. Sorprende descubrir que existe en su obra un cuerpo coherente y estructurado de conocimientos y saberes, teóricos y prácticos, unidos bajo la Conservación.

Ciertamente, la Conservación como disciplina se ha consolidado recientemente, por lo que resulta aún más sorprendente la aportación, que se anticipa a la configuración de la disciplina moderna. Además es bajo ésta, como sus aportaciones a los diferentes campos de la cultura, adquieren la forma de metodología aglutinadora y pionera. Es decir, en Gestoso encontramos un conjunto de reflexiones teóricas que articulan un conocimiento abstracto, que aplica a los ejemplos y casos. En estas prácticas o estrategias, hemos podido encontrar algo más que fragmentos sueltos, los antecedentes de la propia metodología científica de nuestros actuales planes de conservación.

¿Pero qué entendió José Gestoso y Pérez por conservación?

Las palabras que fueron grabadas en el Panteón de llustres Sevillanos recogen esta estrecha relación entre vida y conservación:

"José Gestoso y Pérez gastó su vida en la defensa de los intereses históricos y artísticos de esta ciudad; procurando solícito la conservación de sus tradiciones y monumentos".

Su concepto de conservación parece algo profundo y emocional, un sentimiento que surge ante el rechazo de la destrucción misma. Una experiencia emocional que registra y reacciona frente al paso del tiempo, la destrucción y el olvido. Esa "sensibilidad" se plasma en un doble sentido, en sus reflexiones y en sus prácticas.

Antes de pasar a ellas se puede observar con claridad esa sensibilidad, concretamente en su libro Apuntes del Natural. Leyendas y artículos (Sevilla, 1872), en el que, con un claro tono becqueriano, describe con estas palabras las ruinas del Monasterio de San Isidoro del Campo de Sevilla: 
"El claustro abandonado y sombrío, con sus oscuros sillares corroídos con sus destrozadas pilastras cubiertas por el musgo, con su extenso patio alfombrado de amapolas y jaramagos. ¿Qué conseguiría yo censurando la incuria de unos y la indiferencia de los demás?... La destrucción ha fijado en el monasterio su asiento, el tiempo poco a poco lo va desmoronando y quizás en plazo no lejano veremos al arado abrir sus surcos sobre el sepulcro de Guzmán el Bueno" (Gestoso y Pérez 1879: 26).
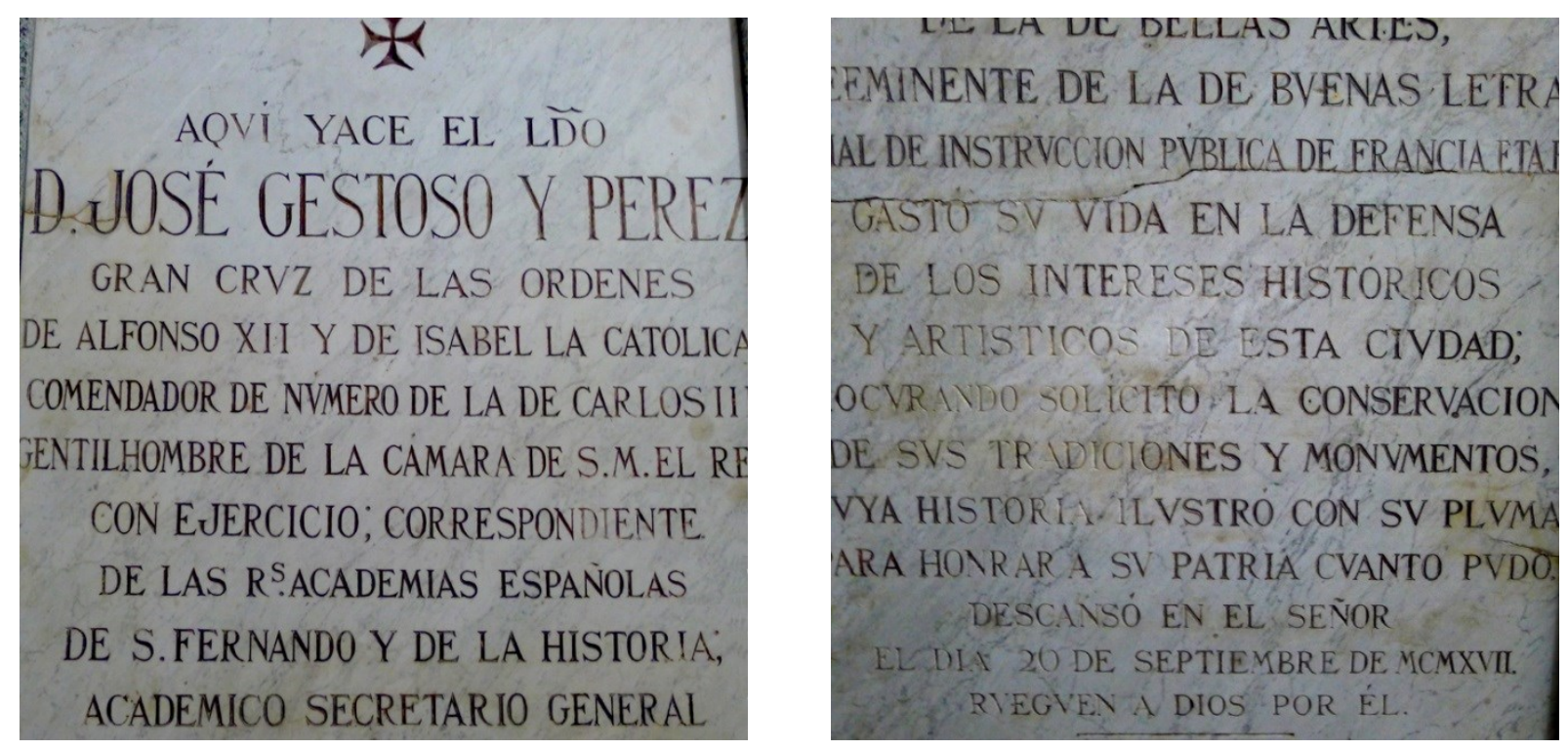

Figuras 3 y 4: Panteón de llustres Sevillanos. Cripta de la Iglesia de la Anunciación. Universidad de Sevilla. Detalles.

\section{La teoría de la Conservación.}

Sustentado en esa sensibilidad, la piedra angular de la conservación, José Gestoso manifiesta claramente cuáles son los objetivos de esta:

- "Salvar del olvido las antiguas memorias de sucesos y cosas que yacen en los archivos y bibliotecas",

- "Llamar la atención pública acerca del abandono en el que algunas yacen",

- "Preocuparse por la pérdida de los testimonios vivos de nuestra cultura pasada",

- "Evitar la desaparición de monumentos, la pérdida de las huellas y pruebas de la memoria" (Gestoso 1872: 8).

En cuanto a la teoría, se pueden apreciar diferentes ideas, conceptos y reflexiones.

Podemos estructurar estas apreciaciones en varios apartados:

- Concepto de monumento.

- Sus reflexiones sobre el concepto de valor e interés.

- Sus reflexiones teóricas sobre el legado material e inmaterial y el valor histórico. 


\subsection{Concepto de monumento}

José Gestoso y Pérez mostró un interés muy amplio hacia las manifestaciones culturales que comprendían desde la conservación de una bandera a un barro vidriado, desde una escultura, a un convento, desde un libro a un documento. De los libros de coro pasó a las pinturas y azulejos polícromos y de estos a los edificios, a los sepulcros y al mobiliario. Hay autores que han visto en esta apertura, y con razón, un anuncio de lo que será el concepto de patrimonio cultural.

Efectivamente, es precisamente en esa apertura donde se observa la evolución de los conceptos antiguos a los nuevos, es decir, del interés por las curiosidades y los monumentos (título que Gestoso y Pérez mantiene en uno de sus libros) hacia otros valores, como son los etnográficos, científicos y técnicos, además de los ya consolidados valores monumentales y arqueológicos. Por ejemplo, de su extensa bibliografía recogemos algunas citas que muestran su preocupación por los libros del Coro de la Catedral de Sevilla (Gestoso y Pérez, 1884:11); por las pinturas de la Casa de Pilato (Gestoso y Pérez, 1884:237), los azulejos polícromos de la Casa del Duque de Alba o la Casa de los Pinelos (Gestoso y Pérez, 1884:139, 143) o bien apela a la conservación de edificios, como el aludido Monasterio de San Isidoro del Campo (Gestoso y Pérez, 1883). Esa renovación de estos conceptos es evidente y ha sido apreciada por otros investigadores, por ejemplo por Adolfo Rodríguez Jurado (1918: 33).

\subsection{Reflexiones sobre su concepto de valor e interés}

Una apreciación nueva es el cambio del sistema de valoración, de uno tradicional a otro más moderno, de uno personal a otro de interés social. Es importante señalar que sustenta el valor en la estima que despierta en los ciudadanos, comprendiendo que son éstos al fin los que hacen posible su trasmisión y conservación. Gestoso se mueve hacia la conservación de interés social. Apela a la competencia municipal y a las autoridades, a las instituciones públicas, prefigurando con ello la gestión del patrimonio del mundo moderno.

\subsection{Sus reflexiones teóricas sobre el legado material e inmaterial y su valor histórico}

La conservación para Gestoso tiene además un fundamento. No responde a criterios más o menos personales, antes bien, descansa en lo que él llama "la verdad histórica". Un nexo invisible une los documentos con el legado material, haciendo imprescindible la relación entre las propias instituciones y los monumentos. Su trabajo en archivos, bibliotecas y museos le permite trazar un puente de conexión entre el legado material y su propio valor histórico. El valor histórico, custodiado en los legajos, en los estratos arqueológicos, en los libros o en los monumentos es otro de los pilares de la conservación.

\section{La práctica de la conservación}

Pero sobre todo, Gestoso articula un plan. La práctica de la conservación es el conjunto de estrategias que hacen posible la transmisión del legado. Y para ello, Gestoso sigue una metodología, cuyo primer paso es la investigación y la búsqueda de documentación en las fuentes archivísticas; le sigue la clasificación -incluida la peritación e informes sobre atribuciones inciertas o falsedades-, para continuar con la exposición, la restauración -si fuera necesaria-y la difusión. 
En éste proceso se puede observar el método científico, un plan aplicado a la propia conservación, como una verdadera aportación pionera.

Hasta aquí se había mostrado a José Gestoso y Pérez en sus distintas facetas, en sus relaciones con las distintas disciplinas. Ahora, bajo la luz de la conservación, se observa claramente su metodología, que comienza con la investigación y continúa con la exposición o apertura a la sociedad, para enlazar con la educación y la difusión.

\subsection{La investigación y búsqueda de documentación}

Es el aspecto más estudiado de la obra de José Gestoso y Pérez. Concretamente fue José Hernández Díaz quién recopiló sus trabajos e hizo hincapié en su perfil de investigador y crítico de arte. Sin embargo, se debe llamar la atención sobre estos estudios guiados por su afán de conservación. Por ejemplo, el que realizó sobre Juan de Valdés Leal (Gestoso y Pérez 1890), donde recoge todo tipo de consultas en los archivos; o los de la vida y obra de Pedro Millán y Pedro de Campaña, con la publicación de datos biográficos, contratos y otros documentos (Gestoso y Pérez, 1899-1908), en donde son frecuentes sus comentarios sobre el estado de conservación y los problemas que conlleva el mantenimiento y cuidado de las obras estudiadas. La búsqueda de la documentación se completa con la clasificación y valoración artística, cuyo resultado será su diccionario de artistas sevillanos (Gestoso y Pérez 1899-1908).

El método de Gestoso se basaba en tomar algunos casos como ejemplo de su metodología. Por ejemplo en algunos monumentos de la provincia de Cádiz, como el Castillo de Luna y la Parroquia Nuestra Señora de la O de Rota (Gestoso y Pérez 1911), o la descripción del Convento de Regla de Chipiona (Gestoso y Pérez 1894), con agudas valoraciones que aún hoy resultan imprescindibles.

\subsection{Catalogaciones, inventarios y guía}

Para Gestoso, lo importante no son los bienes, sino Sevilla, no son los recursos o las riquezas, sino los valores significativos de la ciudad. También en esto parece adelantarse a su tiempo. Su concepto de conservación no queda encerrado en "los objetos o curiosidades", sino que está ligado al de "ciudad". Sevilla para Gestoso es una ciudad compuesta por monumentos y curiosidades que hay que catalogar. Sus bienes muebles e inmuebles requieren de una herramienta eficaz que los describa y los proteja frente a su desaparición. Esa herramienta son los inventarios y los catálogos. Realiza por un lado el catálogo de las pinturas y esculturas del Museo Provincial de Sevilla, y por otro, el catálogo de bienes muebles e inmuebles de la ciudad a partir del propio territorio, construyendo una guía (Gestoso y Pérez 1884). Otros trabajos suyos muy conocidos son el catálogo de bienes muebles de la Catedral o el estudio exhaustivo de su Sacristía y sus bienes muebles (Gestoso y Pérez 1892).

\subsection{Las exposiciones}

Gestoso desarrolla también una amplia labor como comisario de exposiciones, concediendo a la difusión una gran importancia metodológica. Exponer es mostrar al público, es decir, es una de las estrategias fundamentales de la conservación, orientada a la difusión, a la pedagogía y al disfrute. Es un ejemplo claro de hombre ilustrado que vuelve la mirada hacia la sociedad, uniendo la conservación con la ilustración, la pedagogía y la difusión. 
Entre las exposiciones en las que participó cabe destacar las realizadas en Sevilla de "Pintura retrospectiva" (1896), "Bordados" (1898) y "Retratos antiguos" (1910), así como muchas otras de diferentes temas en Madrid (1882), Barcelona (1891, 1892, 1893, 1907, 1910 y 1911), Múnich (1883) y Chicago (1893).

\subsection{La restauración}

La palabra "restauración" tiene para José Gestoso y Pérez un doble sentido: restauración como intervención y restauración como restablecimiento de la memoria perdida. Este es el caso de la restauración de la Cabeza del Rey $D$. Pedro en la que, según sus palabras, "se restaura la memoria del monarca" (Gestoso y Pérez 1985: 7-8)

Fue un teórico y un técnico de la restauración. En primer lugar por sus numerosas alusiones a restauraciones históricas y en segundo por los trabajos que él mismo llevó a cabo. Como historiador de las restauraciones, nos dio a conocer la importancia que tuvo el taller de restauración de la Catedral de Sevilla, que ocupaba la Sala de Descanso y de Rentas. Por ejemplo, así describe una limpieza que se había llevado a cabo en una tabla con la Purísima Concepción de Murillo, de la Sala Capitular con estas palabras:

"Este cuadro se limpió en 1882 sin tocarle en lo más mínimo con los pinceles, y en tal concepto acaso sea el único de los que ejecutó aquel soberano ingenio que se conserva sin repinte ni barnices" (Gestoso y Pérez 1884: 110).

En algunos casos hace una defensa del original:

"Restauraciones que hicieron perder en los originales su valor artístico como el que se refiere a unas estatuas del monumento de Semana Santa" (Gestoso y Pérez 1884:112).

En otros aporta su opinión sobre la restauración, como en el caso de la intervención de una pintura de la Iglesia de San Julián, del pintor Juan Sánchez de Castro:

"El tiempo, la ambición de poderoso magnate por adquirir valiosas joyas y más que nada la ignorancia, el más duro de todos los azotes de nuestras glorias artísticas, han hecho desaparecer las obras del eximio Patriarca (...) si bien lamentando el deplorable estado, pues que por ambos lados de su tercio inferior se encontró bárbaramente aserrada". Y continúa señalando que mejor no se restaure: "lo cual, afortunadamente, no se ha hecho, y creemos que no se debe intentar nunca, si tenemos en cuenta su deplorable estado, pues daría lugar a fantásticos repintes, nunca admisibles" (Gestoso y Pérez 1885: 25).

Se podría señalar una lista o registro de restauradores citados por Gestoso, pero baste recordar a D. Antonio Torrado y D. Rafael Romero Barros en Córdoba, en relación a San Elías y la Virgen de los Plateros de Córdoba, restaurada en 1795 (Gestoso y Pérez 1884: 31).

Como vicepresidente de la Comisión Provincial de Monumentos Históricos y Artísticos, tomó parte activa en numerosas restauraciones llevadas a cabo en edificios emblemáticos, como la Catedral, el Alcázar o la Torre del Oro. Uno de los casos más notables es la restauración del Pendón de la ciudad, que suscitó una cierta polémica ante la decisión del Ayuntamiento de sacar en procesión el original, restaurado por el propio Gestoso. Resulta interesante el efecto que produjo sobre la opinión pública y los medios de comunicación. El pendón quedó finalmente expuesto en el Archivo Municipal y no se volvió a sacar en procesión. 
Otra restauración interesante fue la del León del Alcázar o la polémica defensa de las Murallas de Sevilla.

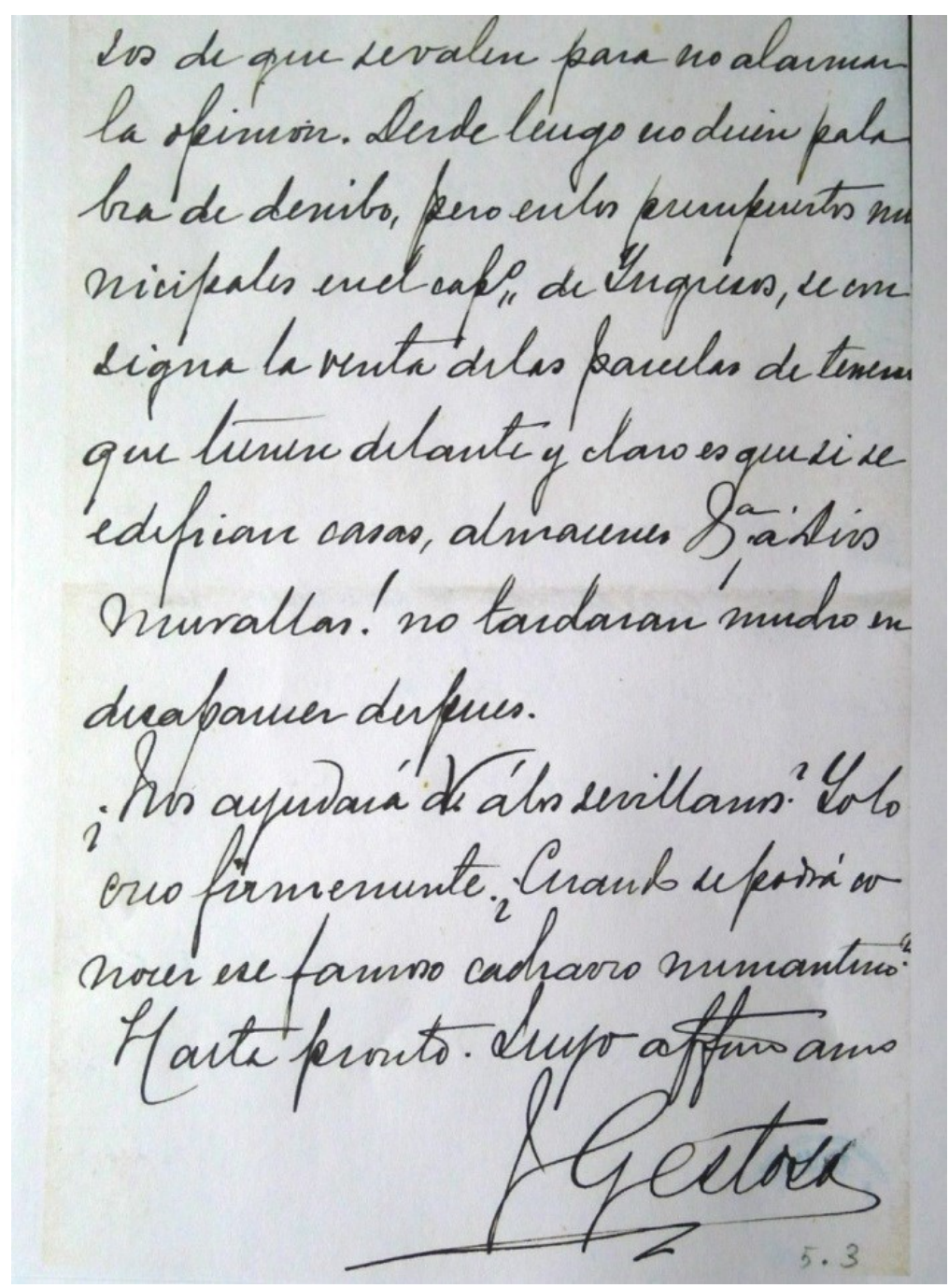

Figuras 5: Imagen de documentos del Archivo Histórico de Andalucía. Carta de José Gestoso y Pérez a J.R. Mélida sobre la conservación de las Murallas de Sevilla. Archivo Histórico de Andalucía. (A.G. A. Misceláneo, 4834.5.3)

Baste reseñar en esta visión general de su contribución como teórico y crítico de la restauración de monumentos su libro "Sevilla monumental y artística", en el que ofrece amplias referencias al estado de conservación de numerosas torres, conventos, parroquias o palacios y otros monumentos sevillanos. Sus alusiones a la acción del tiempo sobre los monumentos son constantes. Son curiosos sus comentarios llamando la atención sobre algunos elementos como, por ejemplo, una piedra romana de mármol en la Torre de Santa Lucía:

“(...) de la incuria de las autoridades para atender a la conservación de los edificios que son el más importante ornamento de esta histórica ciudad, al consentir que se vaya la ruina posesionando de éste, apreciable por tantos títulos y permanezca en poder de un 
particular que en uso de su derecho, podrá derribarlo cuando a bien tenga" (Gestoso y Pérez 1889:136).

En definitiva, Gestoso, hace un cuerpo teórico y crítico sobre Conservación a la par que sobre Historia del Arte (Recio Mir 2008-2009).

Defensor de lo original y buscador del "esplendor prístino" (Gestoso y Pérez 1889: 136, 139), se pronuncia a favor de la eliminación de añadidos barrocos (Gestoso y Pérez 1889: 216) y de la limpieza de la cal de las paredes de muchas iglesias sevillanas (Gestoso y Pérez 1889: 228). También reclama una conservación cuidadosa y por manos peritas. Son muchos los casos donde Ilama la atención en este sentido, por el ejemplo, en la puerta mudéjar del siglo XIV de la Catedral de Sevilla, para la que pide que "procuren su limpieza, siempre que ésta se encomiende a persona perita" (Gestoso y Pérez 1884:102).

\subsection{La difusión}

En cuanto a la difusión, Gestoso y Pérez recurre frecuentemente a la prensa y a los medios de comunicación, otorgándoles el papel de intermediarios entre el patrimonio y los ciudadanos. Su plan incluye la difusión y la información a los ciudadanos sobre la situación del estado de conservación en el que se encuentra el patrimonio. Así se recoge en esta frase:

"A lo largo de su vida, Gestoso se convirtió en un guerrero contra los muchos desmanes, sobre todo arquitectónicos, producidos en su tiempo, dando a conocer, sobre todo a través de sus colaboraciones en prensa, las razones auténticas que enmascaraban las destrucciones, ocultaciones o desnaturalizaciones de obras históricas y artísticas" (Fernández Gómez 2002:165).

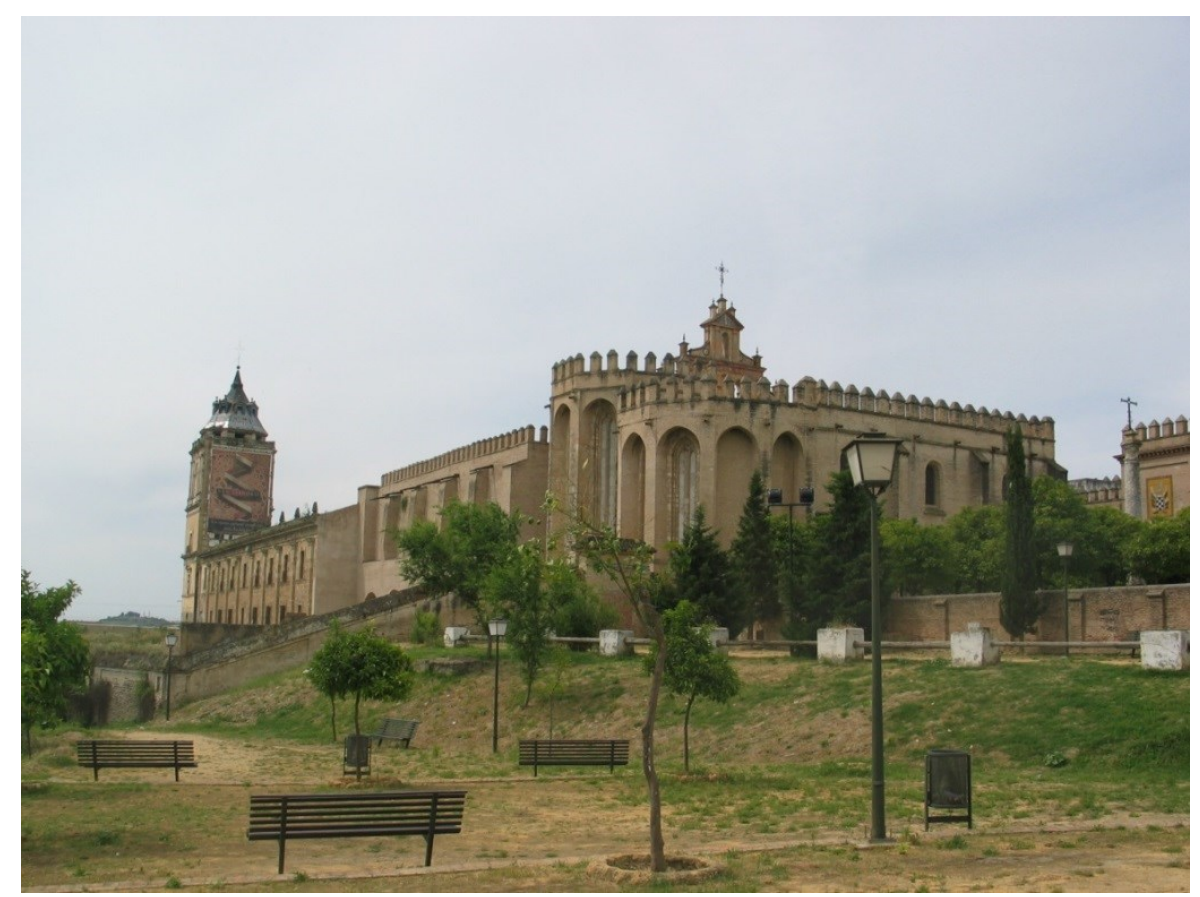

Figura 6: Monasterio San Isidoro del Campo (Sevilla) tras su recuperación en 1992. 


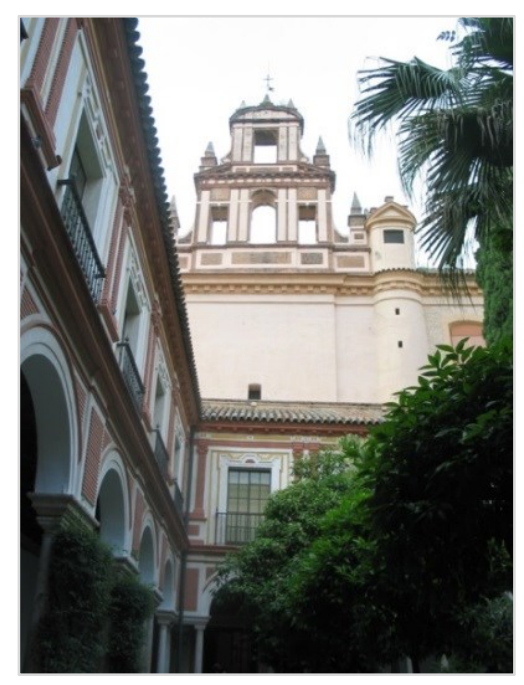

Figura 7: Museo de Bellas Artes de Sevilla. Claustro Grande.

\section{Conclusiones:}

En la actualidad, a la valoración de la fructífera labor de José Gestoso se suma el reconocimiento a sus importantes contribuciones a la Conservación y Restauración como profesional del patrimonio, por los siguientes motivos:

- Por su concepto de conservación, entendido como teoría y práctica.

- Por su manera de articular y organizar la teoría y la práctica, haciéndolas caminar juntas y confluir hacia un objetivo común y único: la conservación.

- Porque entiende por "monumento" un conjunto variado de manifestaciones culturales, en un sentido amplio y diverso que se anticipa en el concepto moderno de bien cultural y patrimonio histórico.

- Por su comprensión del museo como una institución moderna, no como un lugar, sino como un conjunto de bienes organizados, clasificados y abierto al público.

- Por ampliar la conservación y restauración del ámbito del museo a la propia ciudad, y entender que son sus monumentos y sus bienes los que guardan el registro de la memoria.

En cuanto a la aplicación o praxis, también ha quedado clara su metodología, es decir, el conjunto de recursos y estrategias que son los antecedentes de nuestros "planes" de conservación:

- Investigación: Por entender que una de las estrategias para la conservación es la investigación y el estudio. Por comprender que la conservación comienza con la labor de catalogación e inventario, que permite conocer, identificar y clasificar los diferentes bienes de la cultura y del arte, trabajo complejo que configura un eslabón fundamental en la cadena de tutela y protección.

- Exposición: Por defender que la conservación debe dirigirse a la exposición pública. Por su capacidad para organizar exposiciones como estrategias de difusión, entendidas como medios de conservación, en cuanto que acerca un legado cultural a los ciudadanos. 
- Restauración: Por la importancia que tiene en su trabajo la restauración, tanto en un sentido teórico como práctico, tanto dirigida a los bienes muebles como a los inmuebles. También por apelar a la profesionalización de la restauración. Concibe la restauración como una intervención que requiere de peritos, capaces de abordarla con acierto. Apunta que es una operación compleja y crítica, en cuanto que existe una mala praxis o una buena praxis.

- Difusión: Por la importancia que da a los medios de comunicación, y por recurrir a la prensa como elemento de comunicación con la ciudadanía.

\section{Bibliografía}

ALBARDONEDO FREIRE, A. J. (2010). "Precursores del Laboratorio de Arte: Don José Gestoso y sus trabajos patrocinados por el Ayuntamiento de Sevilla". En Estudios de Historia del Arte. Centenario del Laboratorio de Arte (1907-2007), Sevilla: Vicerrectorado de Relaciones Institucionales y Dpto. de $\mathrm{H}^{\mathrm{a}}$ del Arte de la Universidad de Sevilla, Vol. 2, pp. 45-62.

BELTRÁN FORTES, J. (2002). "Descubrimientos arqueológicos en el anfiteatro de Itálica en 1914", Revista de Arqueología, (11), pp. 5-375.

DIDI_HUBERMAN, G. (2009). La imagen superviviente, Historia del arte y tiempo de los fantasmas según Ady Warburg, Madrid: Ed. Abada.

FERNÁNDEZ GÓMEZ, M. (2002-2005). Diccionario de ateneístas de Sevilla, Sevilla: Ateneo de Sevilla, Vol. I.

GESTOSO Y PÉREZ, J. (1883). Apuntes del Natural. Leyendas y artículos. Sevilla, Establecimiento tipográfico de Gironés y Orduña, (Ed. Sevilla, 2001).

-(1884). Pedro Millán. Ensayo biográfico-crítico del escultor sevillano de este nombre, Sevilla: Imprenta de D. Rafael Tarascó.

-(1884). Guía artística de Sevilla. Historia y descripción de los principales monumentos religiosos y civiles, Sevilla: Establecimiento tipográfico de El Orden, (Ed. 2008).

-(1885). Curiosidades antiguas sevillanas. Estudios arqueológicos. Sevilla: Oficina periódico Universal.

-(1889). Sevilla Monumental y Artística. Historia y descripción de todos los edificios notables, religiosos y civiles, que existen actualmente en esta ciudad y noticias de preciosidades artísticas y arqueológicas que en ellos se conservan, Sevilla (Ed. 1984).

-(1890). Biografía del pintor sevillano Juan de Valdés y Leal y estudio de sus obras, Sevilla: Oficina tipográfica de Juan P. Gironés.

-(1892). Historia y descripción de la Sacristía Mayor de la Catedral de Sevilla y de las preciosidades artísticas que en ella se custodian. Sevilla: Imprenta de la Revista de Tribunales.

-(1894). Recuerdos del Monasterio de Nuestra Señora de Regla, Sevilla: Imp. de E. Rasco.

-(1896). Catálogo de las obras que forman la Exposición retrospectiva de la pintura sevillana celebrada en esta ciudad, Sevilla: Tip. de la Región.

-(1899-1908). Ensayo de un diccionario de los artífices que florecieron en Sevilla desde el siglo XIII al XVIII inclusive, Sevilla, "Andalucía moderna", 3 v.

- (1910). Catálogo de la Exposición de Retratos Antiguos celebrada en Sevilla en Abril de MCMX, Madrid: Oficina Tipográfica de Blanco y Negro.

-(1911). Apuntes histórico-descriptivos de la Iglesia y del Castillo de la Villa de Rota, Boletín de la Comisión de Monumentos de la Provincia de Cádiz, t.ll, núm.16, pp. 105-134.

-(1912). Catálogo de Pinturas y Esculturas del Museo Provincial de Sevilla, Madrid: Ed. Lacoste. 
GÓMEZ RAMOS, P. (1975). Catálogo de los tomos manuscritos (papeles varios) del Fondo documental José Gestoso existente en la Biblioteca Capitular Colombina de Sevilla, Memoria de Licenciatura, Universidad de Sevilla.

HERNÁNDEZ DíAZ, J. (1946). Gestoso, investigador y crítico de arte, en Boletín de la Real Academia Sevillana de Buenas Letras, número 72, enero-marzo, pp. 41-49.

LÓPEZ RODRÍGUEZ, J.R. (2010). Historia de los Museos de Andalucía (1500-2000), Sevilla: Universidad de Sevilla.

PLEGUEZUELO HERNÁNDEZ, A. (1995). José Gestoso y la recuperación de la cerámica sevillana (1855-1908). En: Renacimientos: la Cerámica Española en Tiempos de Ruiz de Luna. Ed. Cuenca, pp. 72-89.

RECIO MIR, A. (2009). "El sepulcro neogótico del Cardenal Cienfuegos en la Catedral de Sevilla y la crítica de Gestoso a su "concepto artístico". Laboratorio de Arte, 21 (2008-2009), pp. 219-239.

RODRÍGUEZ JURADO, A. (1918). “Homenaje rendido a la memoria del excelentísimo señor D. José Gestoso y Pérez", en Hernández Díaz, J. (1967): Sevilla: Museo Provincial de Bellas Artes, Madrid: Ministerio de Educación y Ciencia.

RUIZ DE LACANAL RUIZ-MATEOS, M.D. (1994): Conservadores y Restauradores en la Historia de la Conservación y Restauración de Bienes Culturales, Sevilla: Editorial Olimpia.

V.V.A.A. (2011-2012). Gonzalo Bilbao. Fondos del Museo de Bellas Artes de Sevilla. Sevilla: Junta de Andalucía, Consejería de Cultura.

V.V.A.A. (1991). Museo de Bellas Artes, Sevilla: Ediciones Gever.

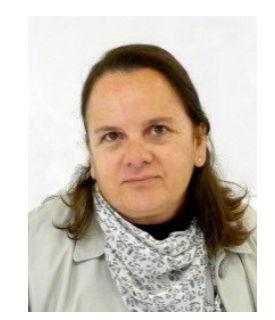

Ma Dolores Ruiz de Lacanal Ruiz-Mateos

mdrmus@us.es

María Dolores Ruiz de Lacanal Ruiz-Mateos es Doctora en Bellas Artes e Historia del Arte y Master en Arquitectura y Patrimonio. Su vida profesional se desarrolla en la Facultad de Bellas Artes de la Universidad de Sevilla, donde imparte la asignatura Teoría e Historia de la Conservación y Restauración de Bienes Culturales y comparte su tiempo entre la investigación y la docencia.

Especialista en esta materia, su primer trabajo se centró en el estudio y evolución de la Conservación y Restauración de los Bienes Culturales en España a través de los profesionales, conservadores y restauradores como protagonistas.

Esta línea de investigación está siendo continuada por el grupo de investigación y desarrollo tecnológico "S.O.S. Patrimonio", que presta un servicio a la sociedad en el ámbito de la conservación y restauración y cuyas actividades comprende desde investigaciones (tesis doctorales, catalogaciones e inventarios, etc.) a trabajos de conservación preventiva y restauración, además de desarrollar proyectos educativos y de difusión.

Entre sus publicaciones destacan: Conservadores y Restauradores en la Historia de la Conservación y Restauración de Bienes Culturales. Estudio del perfil y de la formación (1992), El conservador - restaurador de bienes culturales. Historia de la profesión (1999).

Artículo enviado el 23/08/2012

Artículo aceptado el 13/11/2013 